PROCEEDINGS OF THE

AMERICAN MATHEMATICAL SOCIETY

Volume 140, Number 11, November 2012, Pages 3831-3842

S 0002-9939(2012)11210-9

Article electronically published on March 1, 2012

\title{
PARTIAL LEGENDRE TRANSFORMS OF NON-LINEAR EQUATIONS
}

\author{
PENGFEI GUAN AND D. H. PHONG
}

(Communicated by Chuu-Lian Terng)

\begin{abstract}
The partial Legendre transform of a non-linear elliptic differential equation is shown to be another non-linear elliptic differential equation. In particular, the partial Legendre transform of the Monge-Ampère equation is another equation of Monge-Ampère type. In $1+1$ dimensions, this can be applied to obtain uniform estimates to all orders for the degenerate MongeAmpère equation with boundary data satisfying a strict convexity condition.
\end{abstract}

\section{INTRODUCTION}

The maximum rank and regularity properties of degenerate fully non-linear equations are still largely unexplored, despite their considerable interest for many geometric problems. For example, it is still an unresolved problem raised by Donaldson [9, 10] to determine the precise regularity of geodesics in the spaces of Kähler potentials and of volume forms on a Riemannian manifold. These are given respectively by solutions of a degenerate complex Monge-Ampère equation and an equation introduced by Donaldson [10. Many existence and regularity properties have now been established for these equations (see e.g. [4, 16, 7, 5, 15, 17, 3, 18, 19] and the references therein), but it is not known how close they are to optimal.

In [14, the solution was shown to have a Hessian of maximum rank for several special cases of the degenerate real Monge-Ampère and Donaldson equations, for Dirichlet data satisfying strict convexity conditions. Thus the natural question arises of whether the regularity of solutions of fully non-linear elliptic equations can be established, assuming that it is already known that they have maximum rank.

A potentially useful feature of the maximum rank property is that it allows the use of a partial Legendre transform. In fact, the partial Legendre transform was already exploited by D. Guan 12 in determining geodesics for the space of Kähler potentials on toric varieties and by P. Guan [13] and Rios, Sawyer, and Wheeden 20, 21] in their study of the local regularity of certain degenerate Monge-Ampère equations. A first goal of this paper is to refine their analysis and show that, even though the partial Legendre transform $f$ of a function $u$ is not a local expression of $u$, the partial Legendre transform of an elliptic PDE in $u$ is another PDE in $f$ which is again elliptic. In particular, the original Monge-Ampère equation can be

Received by the editors November 17, 2010 and, in revised form, April 20, 2011.

2010 Mathematics Subject Classification. Primary 35Hxx, 35Jxx; Secondary 58Jxx.

The research of the first author was supported in part by an NSERC Discovery Grant.

The research of the second author was supported in part by National Science Foundation grant DMS-07-57372.

(C)2012 American Mathematical Society 
transformed globally into another dual equation, again of Monge-Ampère type, but which does not seem to have been encountered before in the literature and may be of independent interest (see Theorem 1. (b)). The partial strict convexity properties of one equation are then equivalent to $C^{2}$ estimates for its dual, and one can expect a more general correspondence between bounds for their derivatives. The second goal of this paper is to apply this principle in the simplest case of the $1+1$ real Monge-Ampère equation (which coincides with the $1+1$ Donaldson equation). As a consequence, we obtain $C^{\infty}$ bounds for this equation which depend only on the Dirichlet data, and in particular which remain uniform as the equation degenerates (cf. Theorem 2). We note that in this case, by [12, the solution of the limiting equation is already known to be smooth, so the real interest of the result lies in the uniform validity of the approximation.

\section{LEGENDRE TRANSFORMS}

The main goal of this section is to work out the partial Legendre transforms of fully non-linear elliptic PDE's in some generality. We shall find that, just as in the case of the full Legendre transform, they are given by elliptic PDE's.

2.1. The full Legendre transform. We begin by revisiting the standard Legendre transform. Let $u(x)$ be a strictly convex function on $\mathbf{R}^{n}$. Then $u(x)$ defines a Legendre change of variables

$$
x \rightarrow y=\frac{\partial u}{\partial x}(x) .
$$

Clearly, the Jacobian of this change of variables is $\frac{\partial y_{j}}{\partial x_{k}}=\frac{\partial^{2} u}{\partial x_{j} \partial x_{k}} \equiv u_{j k}$. The strict convexity implies that the map $x \rightarrow y$ from $\mathbf{R}^{n}$ to its image is invertible. The Jacobian of the inverse $y \rightarrow x$ is given by the inverse $u^{j k}$ of the Jacobian $u_{j k}$ of $x \rightarrow y$. Associated to the function $u(x)$ is also its Legendre transform $f(y)$, defined by

$$
f(y)=\langle x, y\rangle-u(x), \quad y=\frac{\partial u}{\partial x}(x) .
$$

Differentiating this relation with respect to $y$ shows that the Legendre change of variables defined by $f(y)$ is the inverse map $y \rightarrow x$,

$$
y \rightarrow x=\frac{\partial f}{\partial y}(y),
$$

and we have the following exact analogues of the earlier formulas for $u, \frac{\partial y_{j}}{\partial x_{k}}=$ $\frac{\partial^{2} f}{\partial x_{j} \partial x_{k}}=u^{j k}$. A partial differential equation of the form $F\left(u_{j k}\right)=0$ can be viewed as a partial differential equation in $f$. Its linearization has principal symbol $\frac{\partial F}{\partial u_{j k}}=f^{j p} f^{k q} \xi_{p} \xi_{q}$. Thus the ellipticity of the equation in $u$ implies the ellipticity of the equation in $f$. In particular, a Monge-Ampère equation for $u$,

$$
\operatorname{det}\left(\frac{\partial^{2} u}{\partial x_{j} \partial x_{k}}\right)=K,
$$

is equivalent to a Monge-Ampère equation for $f$,

$$
\operatorname{det}\left(\frac{\partial^{2} f}{\partial y_{j} \partial y_{k}}\right)=K^{-1} \text {. }
$$


We note that the changes of variables $x \rightarrow y$ and $y \rightarrow x$ in (2.1), (2.3) are unaffected if $u$ and/or $f$ are shifted by independent constants. The relation (2.2) can be viewed as a canonical way of fixing the relative normalization of $f$ and $u$.

2.2. The partial Legendre transform. We come now to the situation of main interest to us, namely partial Legendre transforms of functions $u(x, t)$ which are periodic and satisfy a strict convexity condition in $x$. More specifically, let $e_{i}$ be the basis vectors for $\mathbf{R}^{n}$, that is, $e_{i}$ has component 1 in the $i$-th position, and all its other components are 0 . We consider functions $u(x, t)$ on $\mathbf{R}^{n} \times I, I=(0,1)$, satisfying the periodicity condition

$$
u\left(x+e_{i}, t\right)=u(x, t), \quad x \in \mathbf{R}^{n}, \quad 1 \leq i \leq n,
$$

and the strict convexity condition

$$
\frac{\partial^{2} u}{\partial x_{j} \partial x_{k}}+\delta_{j k}>0
$$

Thus $u$ can also be viewed as a function on $X \times I$, where $X$ is the torus $X=(\mathbf{R} / \mathbf{Z})^{n}$.

We define the following Legendre change of variables:

$$
x \rightarrow y=\left(y_{k}\right), \quad y_{k}=\frac{\partial u}{\partial x_{k}}+x_{k} .
$$

The inverse map $y \rightarrow x$ is well-defined and unique as a map from $\mathbf{R}^{n}$ to $\mathbf{R}^{n}$. To see this, set $v=u+\frac{1}{2}|x|^{2}$, and note that $v \rightarrow \infty$ as $x \rightarrow \infty$. Thus $v$ admits a minimum. After a translation if necessary, we may assume that this minimum is at 0 , and in particular, that $\nabla v$ vanishes there. It suffices then to see that, for any $y$, the equations

$$
\frac{\partial v}{\partial x_{k}}\left(x_{t}\right)=t y_{k}, \quad 0 \leq t \leq 1
$$

can be solved by the method of continuity: the Jacobian $\left(v_{j k}\right)$ is invertible implying openness of the set of $t$ 's for which the equation is solvable. Since $|\nabla v| \rightarrow \infty$ as $|x| \rightarrow \infty$, we also find that $\left|x_{t}\right|$ is bounded and the set of such $t$ 's is also closed.

The maps $x \rightarrow y$ and $y \rightarrow x$ satisfy the following transformation laws:

$$
y_{k}\left(x+e_{i}\right)=y_{k}(x)+\delta_{i k}, \quad x_{k}\left(y+e_{i}\right)=x_{k}(y)+\delta_{i k} .
$$

The condition for $y_{k}$ follows immediately from its definition and the fact that $u(x)$ is periodic. To establish the condition for $x_{k}$, just observe that

$$
y_{k}+\delta_{i k}=\frac{\partial u}{\partial x_{k}}(x)+x_{k}+\delta_{i k}=\frac{\partial u}{\partial x_{k}}\left(x+e_{i}\right)+\left(x+e_{i}\right)_{k}
$$

and the assertion follows by the uniqueness of the inverse map $y \rightarrow x$.

Clearly, the Jacobian of the map $x \rightarrow y$ is given by

$$
\frac{\partial y_{k}}{\partial x_{j}}=\frac{\partial^{2} u}{\partial x_{k} \partial x_{j}}+\delta_{j k} \equiv g_{i j}
$$

Consequently, the Jacobian of the inverse map $y \rightarrow x$ is given by

$$
\frac{\partial x_{j}}{\partial y_{k}}=g^{j k}
$$

All these expressions are periodic and descend to equations on the torus $X$. 
So far, we have discussed the Legendre maps defined by the function $u$. We now define the Legendre transform $f$ of $u$ itself by the following formula:

$$
f(y)=-\frac{1}{2}|x-y|^{2}-u(x), \quad y_{j}=\frac{\partial u}{\partial x_{j}}+x_{j}
$$

for $y \in \mathbf{R}^{n}$. We note that, in view of the transformation laws (2.10) for $x$ and $y$ under period shifts, the function $f(y)$ is actually periodic, and thus can be identified with a function on the torus $X$. Again, the inverse map $y \rightarrow x$ can be viewed as the map associated with the function $f$,

$$
y \rightarrow x_{j}=\frac{\partial f}{\partial y_{j}}+y_{j},
$$

and its Jacobian is given by

$$
\frac{\partial x_{k}}{\partial y_{j}}=\frac{\partial^{2} f}{\partial y_{j} \partial y_{k}}+\delta_{j k}=g^{j k} \equiv h_{j k} .
$$

In particular, the Legendre transform $f$ satisfies the strict convexity condition

$$
D_{y}^{2} f+I>0,
$$

and we still have the relation

$$
\operatorname{det}\left(\frac{\partial^{2} u}{\partial x_{j} \partial x_{k}}+\delta_{j k}\right)=\operatorname{det}^{-1}\left(\frac{\partial^{2} f}{\partial y_{j} \partial y_{k}}+\delta_{j k}\right) .
$$

Consider now the change in variables $\mathbf{R}^{n} \times I \rightarrow \mathbf{R}^{n} \times I$ defined by

$$
(x, t) \rightarrow(y, s), \quad y_{j}=\frac{\partial u}{\partial x_{j}}+x_{j}, \quad s=t .
$$

The Jacobian of the inverse change of variables is given by

$$
t_{s}=1, \quad t_{y_{p}}=0, \quad\left(x_{k}\right)_{y_{p}}=g^{k p}, \quad\left(x_{k}\right)_{s}=-g^{k p} u_{x_{p} t} .
$$

It follows that the rule for differentiating a function $F(x, t)$ with respect to the variables $(y, s)$ is given by

$$
F_{y_{p}}=F_{x_{j}} g^{j p}, \quad F_{s}=-F_{x_{j}} g^{j p} u_{x_{p} t}+F_{t} .
$$

The dependence of the partial Legendre transform on the additional variables $s$ and $t$ is now conveniently described by the following three equations:

$$
\partial_{y_{j}} u_{t}=-\partial_{s} x_{j}, \quad \partial_{s} u_{t}=K(\operatorname{det} g)^{-1}, \quad f_{s}=-u_{t} .
$$

Here $K$ is the $(n+1) \times(n+1)$ determinant $\operatorname{det}\left(D_{x t}^{2} u+I_{x}\right)$,

$$
K=\operatorname{det}\left(D_{x t}^{2} u+I_{x}\right)=u_{t t}(\operatorname{det} g)-G^{j p} u_{t x_{j}} u_{t x_{p}},
$$

where $G^{j p}=(\operatorname{det} g) g^{j p}$ is the matrix of cofactors of the metric $g_{i j}$. To see the first equation, we compute both sides. On the left, we have $\partial_{y_{p}} u_{t}=u_{t x_{j}}\left(x_{j}\right)_{y_{p}}=$ $u_{t x_{j}} g^{j p}$. On the right, we have $-\partial_{s} x_{p}=-\left(-g^{p j} u_{t x_{j}}\right)=g^{p j} u_{t x_{j}}$ also, as required. Next, we apply the rule for differentiation of the previous paragraph and obtain

$$
\partial_{s} u_{t}=-u_{t x_{j}} g^{j p} u_{t x_{p}}+u_{t t}=(\operatorname{det} g)^{-1}\left(u_{t t} \operatorname{det} g-G^{j p} u_{t x_{j}} u_{t x_{p}}\right),
$$

as claimed. Finally, differentiating the defining formula (2.14) for $f$ gives

$$
\partial_{s} f=(y-x) \cdot x_{s}-\left(u_{x} \cdot x_{s}+u_{t}\right)=\left(y-x-u_{x}\right) \cdot x_{s}-u_{t}=-u_{t} .
$$


All three identities in (2.22) have been proved. They imply readily the following two identities, which we also need later:

$$
u_{t x_{j}}=-f_{s y_{k}} h^{k j}, \quad u_{t t}=-f_{s s}+f_{s y_{j}} f_{s y_{k}} h^{j k} .
$$

2.3. The partial Legendre transform of non-linear PDE's. We consider now a fully non-linear equation of the form

$$
F\left(D^{2} u\right)=0
$$

on $X \times I$, where the unknown $u$ is required to satisfy the strict convexity condition $D_{x}^{2} u+I_{x}>0$, and the equation is assumed to be elliptic. We would like to view this equation as an equation for the Legendre transform $f$ of $u$. Note that $f$ is a non-local quantity in $u$. Nevertheless, we have

Theorem 1. Let $X=(\mathbf{R} / \mathbf{Z})^{n}$ be the $n$-dimensional torus, and let $I=(0,1)$. Let $u(x, t)$ be a function on $X \times I$ satisfying the strict convexity condition (2.7). Let $(x, t) \rightarrow(y, s=t)$ be the partial Legendre transform as defined by (2.3), and let $f$ be the partial Legendre transform of the function $u$ as defined by (2.14).

(a) If $F\left(D^{2} u\right)=0$ is a second-order elliptic PDE for $u$, then it can also be viewed as a second-order elliptic PDE for the partial Legendre transform $f$ of $u$.

(b) In particular, u satisfies the Monge-Ampère equation

$$
\operatorname{det}\left(D_{x, t}^{2} u+I_{x}\right)=K
$$

if and only if its partial Legendre transform $f$ satisfies the following equation on $X \times I$ also of Monge-Ampère type:

$$
\frac{\partial^{2} f}{\partial s^{2}}+K \operatorname{det}\left(D_{y}^{2} f+I\right)=0
$$

Proof. From the discussion in the preceding section, the partial Legendre transform $f$ of $u$ is a well-defined function on $X \times I, \partial_{j} \partial_{k} u+\delta_{j k}$ are given by the inverse of the matrix $\partial_{j} \partial_{k} f+\delta_{j k}$, and $u_{t x_{j}}$ and $u_{t t}$ are given by the expressions (2.26) in the second derivatives of $f$. Thus the equation $F\left(D^{2} u\right)=0$ is automatically a second-order non-linear equation for $f$. To verify the ellipticity of the equation viewed as an equation for $f$, we work out first the linearized operator of $F\left(D^{2} u\right)$, keeping variations in $\delta u$,

$$
\delta F=\frac{\partial F}{\partial u_{t t}}(\delta u)_{t t}+\frac{\partial F}{\partial u_{t x_{j}}}(\delta u)_{t x_{j}}+\frac{\partial F}{\partial u_{x_{j} x_{k}}}(\delta u)_{x_{j} x_{k}} .
$$

We need to express this quantity in terms of the derivatives of $\delta f$. In view of the expression (2.26) for $\delta u_{t t}$ and $\delta u_{t x_{j}}$, we have

$$
\begin{aligned}
\delta u_{t t} & =-\delta f_{s s}+2 \delta f_{s y_{j}} f_{s y_{k}} h^{j k}-f_{s y_{j}} f_{s y_{k}} \delta f^{j k}, \\
\delta u_{t x_{j}} & =-\delta f_{s y_{k}} h^{k j}+f_{s y_{j}} \delta f^{j k},
\end{aligned}
$$

where the indices are raised or lowered using the metric $h_{j k}$. Thus we have

$$
\begin{aligned}
\delta F= & -\frac{\partial F}{\partial u_{t t}} \delta f_{s s}+\left(-\frac{\partial F}{\partial u_{t x_{j}}}+2 \frac{\partial F}{\partial u_{t t}} f_{s y_{j}}\right) h^{j k} \delta f_{s y_{k}} \\
& +\left(-\frac{\partial F}{\partial u_{t t}} f_{s y_{j}} f_{s y_{k}}+\frac{\partial F}{\partial u_{t x_{j}}} f_{s y_{k}}-\frac{\partial F}{\partial u_{x_{j} x_{k}}}\right) \delta f^{j k} .
\end{aligned}
$$


This means that if $\tau$ and $\xi_{j}$ are respectively the variables dual to $t$ and $x_{j}$, the symbol $\sigma(\tau, \xi)$ of the linearized operator is given by

$$
\begin{aligned}
\sigma(\tau, \xi)= & \frac{\partial F}{\partial u_{t t}} \tau^{2}+\left(\frac{\partial F}{\partial u_{t x_{j}}}-2 \frac{\partial F}{\partial u_{t t}} f_{s y_{j}}\right) h^{j k} \tau \xi_{k} \\
& +\left(\frac{\partial F}{\partial u_{t t}} f_{s y_{j}} f_{s y_{k}}-\frac{\partial F}{\partial u_{t x_{j}}} f_{s y_{k}}+\frac{\partial F}{\partial u_{x_{j} x_{k}}}\right) \xi_{j} \xi_{k} .
\end{aligned}
$$

We shall show that $\sigma(\tau, \xi)$ is positive definite if the original equation $F\left(D^{2} u\right)=0$ is elliptic. Introduce the variable $\eta_{j} \equiv h^{j k} \xi_{k}$ for convenience. Then completing the square in $\tau$ gives

$$
\begin{aligned}
\sigma(\tau, \xi)= & {\left[\tau \sqrt{\frac{\partial F}{\partial u_{t t}}}+\left(\frac{1}{2} \frac{\partial F}{\partial u_{t x_{j}}}-\frac{\partial F}{\partial u_{t t}} f_{s y_{j}}\right) \frac{\eta_{j}}{\sqrt{\frac{\partial F}{\partial u_{t t}}}}\right]^{2} } \\
& +\frac{1}{4 \frac{\partial F}{\partial u_{t t}}}\left[4 \frac{\partial F}{\partial u_{t t}} \frac{\partial F}{\partial u_{x_{j} x_{k}}} \eta_{j} \eta_{k}-\left(\frac{\partial F}{\partial u_{t x_{j}}} \eta_{j}\right)^{2}\right] .
\end{aligned}
$$

To prove part (a) of the theorem, it suffices then to show that the second term on the right is strictly positive for $\eta \neq 0$ when $F$ is elliptic. Since the symbol of the linearized operator when the unknown is $u$ is given by

$$
\frac{\partial F}{\partial u_{t t}} \tau^{2}+\frac{\partial F}{\partial u_{t x_{j}}} \tau \xi_{j}+\frac{\partial F}{\partial u_{x_{j} x_{k}}} \xi_{j} \xi_{k}
$$

its ellipticity does imply the positivity of the second term on the right hand side of (2.34). Part (a) is proved.

Part (b) follows immediately from the identities in (2.22),

$$
f_{s s}=-\partial_{s} u_{t}=-K(\operatorname{det} g)^{-1}=-K \operatorname{det}\left(D_{y}^{2} f+I\right) .
$$

The proof of Theorem 1 is complete.

We conclude this section with a few remarks.

- Part (b) of Theorem 1 can be viewed as a refinement of several earlier results in the literature using the partial Legendre transform: when $K=0$, it reproduces the result of D. Guan [12. For general $K$ and when the considerations are local (instead of on a torus as here), then P. Guan [13] in dimension $n=1$ and RiosSawyer-Wheeden 20] for general dimension $n$ have shown that the coordinates $x_{j}$, viewed as functions of $(y, s)$, satisfy the following elliptic, non-linear system of equations:

$$
\partial_{s}^{2} x_{j}+\frac{\partial}{\partial y_{j}}\left(K \operatorname{det}\left(\frac{\partial x_{k}}{\partial y_{m}}\right)\right)=0, \quad 1 \leq j \leq n .
$$

This system of equations follows immediately from differentiation of the equation (2.29) with respect to $y_{j}$.

- The presence of the background symmetric form $\delta_{j k}$ is very similar to the presence of the Kähler form $\omega$ for the complex Monge-Ampère equation $\left(\omega+\frac{i}{2} \partial \bar{\partial} \phi\right)^{n}=$ $F \omega^{n}$.

- The correspondence between an equation in $u$ and its "dual" equation in $f$ can provide non-trivial information. For example, it is not evident that the equation (2.29) admits smooth solutions for given Dirichlet data, even when $K$ is a strictly positive constant. On the other hand, the existence of such solutions is an immediate consequence of the existence of smooth solutions for the dual equation (2.28), 
which can be established by the theory of Caffarelli-Nirenberg-Spruck [8], with the improved barrier arguments of B. Guan [11].

Similarly, lower bounds for $D_{x}^{2} u+I$ are equivalent to upper bounds for $D_{y}^{2} f+I$ and vice versa, and the problems of partial $C^{2}$ estimates and partial strict convexity are in this sense "dual". For example, the $C^{2}$ estimates for the original MongeAmpère equation (2.28) can be established by traditional methods as in 8 or as a consequence of the convexity results for the dual equation (2.29), using for example the recent results of Bian and Guan [1, 2.

- Beyond the Monge-Ampère equation, the partial Legendre transforms of Hessian equations may be of interest. By (2.26),

$$
D_{x, t}^{2} u+I=\left(\begin{array}{cccc}
-\tilde{K}(\operatorname{det} \tilde{g})^{-1} & -\tilde{u}_{s y_{1}} \lambda_{1}^{-1} & \cdots & -\tilde{u}_{s y_{n}} \lambda_{n}^{-1} \\
-\tilde{u}_{s y_{1}} \lambda_{1}^{-1} & \lambda_{1}^{-1} & \cdots & 0 \\
\cdots & & & \\
-\tilde{u}_{s y_{n}} \lambda_{n}^{-1} & 0 & \cdots & \lambda_{n}^{-1}
\end{array}\right),
$$

where the $\lambda_{i}$ are the eigenvalues of $\tilde{g}=\left(\tilde{u}_{i j}+\delta_{i j}\right)$, and we have denoted for convenience all quantities associated with the partial Legendre transform by $\mathrm{a}^{\sim}$ (e.g. $f$ is now denoted $\tilde{u}$, and $\tilde{K}$ is the Monge-Ampère determinant of $D_{y, s}^{2} \tilde{u}+I$ ). The standard formula for the $k$-th symmetric function $\sigma_{k}$ of the eigenvalues of a matrix is

$$
\sigma_{k}(V)=\frac{1}{k !} \sum \delta_{j_{1} \cdots j_{k}}^{i_{1} \cdots i_{k}} v_{i_{1} j_{1}} \cdots v_{i_{k} j_{k}} .
$$

We apply this formula to the symmetric function $\hat{\sigma}$ of the $(n+1)$-dimensional matrix $D_{x, t}^{2} u+I$. We find

$$
\hat{\sigma}_{k}\left(D_{x, t}^{2} u+I\right)=\sigma_{k}\left(\tilde{g}^{-1}\right)-\sum_{i_{1} \cdots i_{k-1}} \lambda_{i_{1}}^{-1} \cdots \lambda_{i_{k-1}}^{-1}\left(\sum_{i_{j}=1}^{k-1} \tilde{u}_{s y_{i_{j}}}^{2} \lambda_{i_{j}}^{-1}+\tilde{K}(\operatorname{det} \tilde{g})^{-1}\right) .
$$

From here, it is easily seen that the Laplace equation $u_{t t}+\Delta u=K_{1}$ gets transformed into

$$
\tilde{K}+K_{1} \operatorname{det} \tilde{g}=\sigma_{n-1}(\tilde{g}) .
$$

For general $k$, the equation $\hat{\sigma}_{k}(u)=K_{k}$ gets transformed into

$$
K_{k}=\frac{\sigma_{n-k}(\tilde{g})}{\sigma_{n}(\tilde{g})}-\sum_{i_{1} \cdots i_{k-1}} \lambda_{i_{1}}^{-1} \cdots \lambda_{i_{k-1}}^{-1}\left(\sum_{i_{j}=1}^{k-1} \tilde{u}_{s y_{i_{j}}}^{2} \lambda_{i_{j}}^{-1}+\tilde{K}(\operatorname{det} \tilde{g})^{-1}\right) .
$$

We note that when $k=n+1$, the above identity recovers (2.29).

\section{The $1+1$ Monge-Ampère equation}

In this section, we consider more specifically the case $n=1$ of the Monge-Ampère equation. In this case, the equation becomes the following equation on $X \times I$ :

$$
u_{t t}\left(1+u_{x x}\right)-u_{x t}^{2}=\varepsilon,
$$

where $X=\mathbf{R} / \mathbf{Z}$ is a circle and $\varepsilon>0$ is a constant. The solution $u$ is required to satisfy $D_{x t}^{2} u+I_{x} \geq 0$, and we impose the Dirichlet condition $u(t, 0)=u^{0}(x)$, $u(t, 1)=u^{1}(x)$, with $u^{i} \in C^{\infty}(X)$ satisfying the strict convexity condition,

$$
u_{x x}^{i}+1 \geq \lambda
$$


for $i=0,1$ and $\lambda>0$ a constant. We have

Theorem 2. Let $u$ be the solution of the equation (3.1) on $X^{1} \times I$, with $D_{x t}^{2} u+I_{x} \geq$ 0 and smooth Dirichlet data satisfying (3.2). Then for any non-negative integer $N$, there exists $M(N)$ and a constant $C_{N}$ depending only on $\lambda>0$ and the $C^{M(N)}$ norms of the Dirichlet data $u^{0}, u^{1}$ so that

$$
\sum_{a+b \leq N}\left\|\partial_{x}^{a} \partial_{t}^{b} u\right\|_{C^{0}\left(X^{1} \times I\right)} \leq C_{N} .
$$

In particular, the constants $C_{N}$ are independent of $\varepsilon$.

The rest of this section is devoted to the proof of Theorem 2. To apply the partial Legendre transform as in $\S 2$, we need the strict partial convexity of $u$. This follows from Theorem 1 of [14]. But since the present case is particularly simple, we can supply the short proof for the convenience of the reader:

Lemma 1. Let $u(x, t)$ be the solution of the equation (3.1), as specified in the statement of Theorem 2, Then $u_{x x}(x, t)+1 \geq \lambda$ for all $x \in X$, where $\lambda$ is the lower bound for the boundary data in (3.2).

Proof of Lemma 1. Let $\lambda_{0}=\min _{(x, t) \in X \times \bar{I}}\left(u_{x x}+1\right)$, and set $\varphi(x, t)=u_{x x}+1-\lambda_{0}$. We establish a strong maximum principle for $\varphi$. If $\varphi$ vanishes on the boundary, the lemma is proved. We shall show that in a neighborhood of any interior zero of $\varphi$, $\varphi$ satisfies an elliptic differential inequality equation of the form

$$
F^{i j} \varphi_{i j} \leq C|\nabla \varphi| \text {. }
$$

Here we have denoted by $F$ the function $F\left(D_{x t}^{2} u+I_{x}\right)$ with $F(M)=\operatorname{det}\left(M_{i j}\right)$, for $M$ any symmetric $2 \times 2$ matrix, and $F^{i j}=\frac{\partial F}{\partial M_{i j}}$. The constant $C$ is required to be independent of the point $(x, t)$ but may depend on everything else. By the strong maximum principle, this would imply that $\varphi$ vanishes in a neighborhood of any interior zero. If the set of such zeroes is not empty, then $\varphi$ vanishes identically. By continuity $\varphi$ would again vanish on the boundary, and the lemma is proved in all cases.

The equation (3.1) can be written as $F\left(D_{x t}^{2} u+I_{x}\right)=\varepsilon$. Differentiating the equation successively gives

$$
F^{i j} u_{i j x}=0, \quad F^{i j} u_{i j x x}+F^{i j, k l} u_{i j x} u_{k l x}=0 .
$$

Thus $F^{i j} \varphi_{i j}=-F^{i j, k l} u_{i j x} u_{k l x}$, and more explicitly,

$$
F^{i j} \varphi_{i j}=-2 u_{t t x} u_{x x x}-2 u_{x x t}^{2}=-2 u_{t t x} \varphi_{x}-2 \varphi_{x}^{2} .
$$

The inequality (3.4) follows.

Let $f(y, s)$ be now the partial Legendre transform of $u$, as defined in section 2.2. When $n=1$, the equation for $f$ simplifies to

$$
L f \equiv f_{s s}+\varepsilon f_{y y}=-\varepsilon \quad \text { on } X \times I,
$$

and $f(y, 0)$ and $f(y, 1)$ are given by the Legendre transforms $f^{0}(y)$ and $f^{1}(y)$ of the functions $u^{0}(x)$ and $u^{1}(x)$. General linear elliptic theory says that any derivative of $f$ can be bounded in terms of the boundary data and the ellipticity constant $\varepsilon$. However, we require estimates which are uniform as $\varepsilon \rightarrow 0$, and such estimates do not seem to have been written down in the literature. We provide below a brief and explicit derivation of estimates uniform in $\varepsilon$, exploiting the simple form of the 
equation and of the boundary in the present case. More precisely, we shall establish the following lemma:

Lemma 2. Consider the Dirichlet problem for the Laplacian $L$ in (3.7) with $\varepsilon$ a constant satisfying $0<\varepsilon<1$. Then for any $m, k$ we have

$$
\left\|\partial_{y}^{m} \partial_{s}^{k} f\right\|_{C^{0}(\tilde{X} \times I)} \leq C_{m, k}
$$

where the $C_{m, k}$ are constants which depend only on the Dirichlet data (and on $m$ and $k$ ). In particular, the $C_{m, k}$ are independent of $\varepsilon$.

Proof. Clearly $\partial_{y}^{m} \partial_{s}^{k} f$ satisfies the same Laplace equation. By the maximum principle, we have then

$$
\left\|\partial_{y}^{m} \partial_{s}^{k} f\right\|_{C^{0}(\tilde{X} \times I)}=\left\|\partial_{y}^{m} \partial_{s}^{k} f\right\|_{C^{0}(\tilde{X} \times \partial I)} .
$$

We shall show that the right hand sides can be estimated in terms of the Dirichlet data alone, for arbitrary $m$ and $k=0$ or $k=1$. Assuming this, it follows that the left hand side is also bounded in terms of the Dirichlet data alone for these values of $m$ and $k$. Since the equation implies that $\partial_{y}^{m} \partial_{s}^{k} f=-\varepsilon \partial_{y}^{m+2} \partial_{s}^{k-2} f$ for $k \geq 2$, it follows that uniform bounds for $\partial_{y}^{m} \partial_{s}^{k} f$ for arbitrary $k$ follow from the special cases $k=0$ and $k=1$, and the lemma would be proved.

We return to the proof of bounds for $\left\|\partial_{y}^{m} \partial_{s}^{k} f\right\|_{C^{0}(\tilde{X} \times \partial I)}$ when $k=0$ or $k=1$. When $k=0$, they are obvious, so we concentrate on the case $k=1$. Let $\tilde{f}$ be a function with the same boundary values as $f$ (e.g., $\tilde{f}=t f(y, 1)+(1-t) f(y, 0))$. Set

$$
w(y, s)=-A s^{2}+B s
$$

for constants $A, B>0$, which we shall choose in a moment. Since $L\left(\partial_{y}^{m} f\right)=0$, we have

$$
L\left(\partial_{y}^{m}(f-\tilde{f})\right)-L\left(\partial_{y}^{m} \tilde{f}\right) \geq c_{1},
$$

where $c_{1}$ is a constant depending only on the Dirichlet data and independent of $0<\varepsilon<1$. On the other hand,

$$
L w=-2 A,
$$

so we can choose $A$ large enough so that $L w \leq L\left(\partial_{y}^{m}(f-\tilde{f})\right)$ on $\tilde{X} \times I$. Now $\partial_{y}^{m}(f-\tilde{f})$ vanishes identically on the boundary, so if we choose $B$ large enough so that $w \geq 0$ everywhere, we shall also have $\partial_{y}^{m}(f-\tilde{f}) \leq w$ on the boundary. Thus, by the comparison principle, $\partial_{y}^{m}(f-\tilde{f}) \leq w$ on $\tilde{X} \times I$. Since both of these functions vanish at $s=0$, we obtain

$$
\partial_{s} \partial_{y}^{m} f \leq-2 A s+B+\partial_{s} \partial_{y}^{m} \tilde{f}
$$

which is an upper bound for $\partial_{s} \partial_{y}^{m} f$. Applying the argument to $-f$ instead of $f$, we obtain a lower bound for $\partial_{s} \partial_{y}^{m} f$ at the boundary points $(y, 0)$. Since the argument at the boundary points $(y, 1)$ is identical, the proof of the lemma is complete.

Next, we show that $C^{N}$ bounds for $x$ (viewed as a function of $(y, s)$ ), together with a strict partial convexity bound, imply $C^{M}$ bounds for the original function $u$. 
Lemma 3. For any non-negative integer $M$, we have

$$
\sum_{m+b \leq M}\left\|\partial_{x}^{m} \partial_{t}^{b} u\right\|_{C^{0}\left(X^{1} \times I\right)} \leq C_{M},
$$

where $C_{M}$ is a constant depending only on the Dirichlet data $u^{0}, u^{1}$, the lower bound $\lambda>0$, and the $C^{0}$ norm of a finite number $N(M)$ of spatial derivatives $\partial_{y}^{m} x$ of the function $x$.

Proof of Lemma 3. First, we show that bounds for $\partial_{y}^{m} x$ imply bounds for $\partial_{x}^{a} u$. This is an easy consequence of the following formula, which is itself a consequence of the chain rule established in the previous section:

$$
\partial_{y}^{m} x=-\frac{1}{\left(u_{x x}+1\right)^{m}} \partial_{x}^{m+1} u+\frac{P\left(u, \cdots, \partial_{x}^{m} u\right)}{\left(u_{x x}+1\right)^{2 m-1}},
$$

where $P$ is a generic notation for a polynomial in all its entries for all $m \geq 2$.

Now for $m=2$, the bounds of $\partial_{x}^{m} u$ in terms of boundary data alone are a special case of the $C^{2}$ estimates for the Dirichlet problem for the Monge-Ampère equation 8. Note that these bounds do not require a strictly positive lower bound for the Monge-Ampère determinant and thus give bounds which are uniform in $\varepsilon$ in our case.

Assume that bounds depending only on the Dirichlet data and a strictly positive lower bound $\lambda$ for $u_{x x}+1$ have been established for $m$. In view of the above formula for $\partial_{y}^{m} x$, it follows that such bounds for $\partial_{x}^{m+1} u$ reduce to such bounds for $\partial_{y}^{m} x$ on $\tilde{X} \times I$. By the maximum principle for $\partial_{y}^{m} x$, this reduces in turn to bounds for $\partial_{y}^{m} x$ only on the boundary. But the same formula (3.15) for higher derivatives above shows that $\partial_{y}^{m} x$ on the boundary $\tilde{X} \times \partial I$ is determined completely by the boundary data. This establishes the desired bounds for $\left\|\partial_{x}^{a} u\right\|_{C^{0}(X \times I)}$.

Next, we consider mixed derivatives of the form $\partial_{x}^{a} \partial_{t} u$. It is again easy to establish the following general formula linking $\partial_{y}^{m} \partial_{s} x$ and $\partial_{x}^{a} \partial_{t} u$ for all $m \geq 0$ :

$$
\partial_{y}^{m} \partial_{s} x=-\frac{\partial_{x}^{m+1} \partial_{t} u}{\left(u_{x x}+1\right)^{m+1}}+\frac{P\left(u, \cdots, \partial_{x}^{m+2} u, \partial_{t} u, \cdots, \partial_{x}^{m} \partial_{t} u\right)}{\left(u_{x x}+1\right)^{2 m+1}},
$$

where $P$ is again a polynomial in all its entries. In view of Lemma 2 the left hand side can be bounded uniformly in terms of the Dirichlet data and the lower bound $\lambda$ for $u_{x x}+1$. Thus the formula implies that $\partial_{x}^{m+1} \partial_{t} u$ can be similarly bounded if $\partial_{x}^{m} \partial_{t} u$ is. Since $u_{x t}$ is bounded by the Dirichlet data in view of the $C^{2}$ estimates, we obtain by induction the uniform boundedness of $\partial_{x}^{m} \partial_{t} u$ for all $m$.

Finally, by differentiating the original Monge-Ampère equation, we can show inductively on the number $b$ of $t$ derivatives in $\partial_{x}^{a} \partial_{t}^{b} u$ that they are in turn bounded. First, we show this for $b=2$. Differentiating the equation $m$ times with respect to $x$ gives

$$
\partial_{x}^{m} u_{t t}=\frac{1}{u_{x x}+1} P\left(\partial_{x}^{2} u, \cdots, \partial_{x}^{m+2} u, \partial_{t} \partial_{x} u, \cdots, \partial_{t} \partial_{x}^{m+1} u, \partial_{t}^{2} u, \cdots, \partial_{t}^{2} \partial_{x}^{m-1} u\right)
$$

with $P$ a polynomial in all its entries. Since $\partial_{x}^{m} u_{t t}$ is bounded for $m=0$ by the $C^{2}$ estimates, and $\partial_{x}^{a} u, \partial_{x}^{a} \partial_{t} u$ are now known to be bounded, the formula allows us to show by induction on $m$ that $\partial_{x}^{m} \partial_{t}^{2} u$ is bounded. Next, assume that $\partial_{x}^{m} \partial_{t}^{b} u$ is bounded for an integer $b \geq 2$ and all non-negative integers $m$. We shall show that 
this remains true if $b$ is replaced by $b+1$. Differentiating the equation $b-1$ times gives

$$
\partial_{t}^{b+1} u=\frac{1}{u_{x x}+1} P\left(\partial_{x} \partial_{t}, \cdots, \partial_{x} \partial_{t}^{b} u, \partial_{x}^{2} \partial_{t} u, \cdots, \partial_{x}^{2} \partial_{t}^{b} u\right),
$$

where $P$ is a polynomial in all its entries. This shows that $\partial_{x}^{m} \partial_{t}^{b+1} u$ is bounded for $m=0$. Thus it suffices to show that if $\partial_{x}^{\ell} \partial_{t}^{b+1} u$ is bounded for all non-negative integers $\ell \leq m$, then the same is true if $m$ is replaced by $m+1$. But this follows readily by differentiating the previous formula,

$$
\partial_{x}^{m+1} \partial_{t}^{b+1} u=\partial_{x}^{m+1}\left\{\frac{1}{u_{x x}+1} P\left(\partial_{x} \partial_{t}, \cdots, \partial_{x} \partial_{t}^{b} u, \partial_{x}^{2} \partial_{t} u, \cdots, \partial_{x}^{2} \partial_{t}^{b} u\right)\right\} .
$$

Since the right hand side involves terms with at most $b$ derivatives in $t$, and all derivatives in $x$ of such expressions are bounded, the desired bound for $\partial_{x}^{m+1} \partial_{t}^{b+1} u$ follows.

Putting together Lemmas 2-4, we obtain Theorem 2 ,

\section{REFERENCES}

1. Bian, B. and P. Guan, "A microscopic convexity principle for nonlinear partial differential equations", Inventiones Math. 177 (2009) 307-335. MR 2511744 (2010i:35086)

2. Bian, B. and P. Guan, "A structural condition for microscopic convexity principle", Discrete and Continuous Dynamical Systems, 28 (2010) 789-807. MR2644765 (2011c:35216)

3. Blocki, Z., "On geodesics in the space of Kähler metrics", to appear in the Proceedings of the "Conference on Geometry", dedicated to Shing-Tung Yau, Warsaw, 2009.

4. Chen, X.X., "The space of Kähler metrics", J. Differential Geom. 56 (2000) 189-234. MR.1863016 (2003b:32031)

5. Chen, X.X. and W. He, "The space of volume forms", arXiv:0810.3880

6. Chen, X.X. and S. Sun, "Space of Kähler metrics (V): Kähler quantization", arXiv:0902.4149

7. Chen, X.X. and G. Tian, "Geometry of Kähler metrics and foliations by holomorphic discs". Publ. Math. Inst. Hautes Études Sci. No. 107 (2008) 1-107. MR2434691 (2009g:32048)

8. Caffarelli, L., L. Nirenberg, and J. Spruck, "The Dirichlet problem for non-linear second-order elliptic equations I. Monge-Ampère equations", Comm. Pure Appl. Math. XXXVII (1984) 369-402. MR739925 (87f:35096)

9. Donaldson, S.K., "Symmetric spaces, Kähler geometry, and Hamiltonian dynamics", Amer. Math. Soc. Transl. Ser. 2, 196, Amer. Math. Soc., Providence, RI, 1999, 13-33. MR1736211 (2002b:58008)

10. Donaldson, S.K., "Nahm's equations and free boundary problems", in The Many Facets of Geometry, 71-91, Oxford Univ. Press, Oxford, 2010.

11. Guan, B., "The Dirichlet problem for Hessian equations on Riemannian manifolds". Calc. Var. Partial Differential Equations 8 (1999) 45-69. MR1666866 (99k:58191)

12. Guan, D., "On modified Mabuchi functional and Mabuchi moduli space of Kähler metrics on toric bundles", Math. Res. Lett. 6 (1999), no. 5-6, 547-555. MR1739213 (2001b:32042)

13. Guan, P., "Regularity of a Class of Quasilinear Degenerate Elliptic Equations", Advances in Mathematics, Vol. 132 (1997) 24-45. MR1488238(99a:35068)

14. Guan, P. and D.H. Phong, "A maximum rank problem for degenerate elliptic fully non-linear equations". To appear in Math. Ann., arXiv:1009.3975

15. He, W., "The Donaldson equation", arXiv:0810.4123

16. Phong, D.H. and J. Sturm, "The Monge-Ampère operator and geodesics in the space of Kähler potentials", Inventiones Math. 166 (2006) 125-149. MR2242635 (2007h:32036)

17. Phong, D.H. and J. Sturm, "Test configurations for K-stability and geodesic rays", J. Symplectic Geom. 5 (2007) no. 2, 221-247. MR2377252(2009a:32033) 
18. Phong, D.H. and J. Sturm, "Lectures on stability and constant scalar curvature", Current Developments in Mathematics, 2007, 101-176, International Press, Somerville, MA, 2009. MR2532997 (2010j:32042)

19. Phong, D.H. and J. Sturm, "The Dirichlet problem for degenerate complex Monge-Ampère equations", Comm. Anal. Geom 18 (2010) no. 1, 145-170. MR.2660461.

20. Rios, C., E. Sawyer and W. Wheeden, "A higher-dimensional partial Legendre transform, and regularity of degenerate Monge-Ampère equations". Adv. Math. 193 (2005) no. 2, 373-415. MR2137289 (2006f:35099)

21. Rios, C., E. Sawyer and W. Wheeden, "Regularity of subelliptic Monge-Ampère equations". Adv. Math. 217 (2008) no. 3, 967-1026. MR.2383892 (2009k:35086)

Department of Mathematics, McGill University, Montreal, Quebec H3A 2K6, CANADA

E-mail address: guan@math.mcgill.ca

Department of Mathematics, Columbia University, New York, New York 10027

E-mail address: phong@math.columbia.edu 